\title{
Randomized Reductions and the Topology of Conjectured Classes of Uniquely Hamiltonian Graphs
}

\author{
Robert D. Barish ${ }^{1, a)} \quad$ AkIra Suyama $^{1, b)}$ \\ Received: December 31, 2019, Accepted: September 10, 2020
}

\begin{abstract}
We utilize the hardness of the Unambiguous-SAT problem under randomized polynomial time reductions (Valiant \& Vazirani; Theoret. Comput. Sci., Vol.47, 1986) to probe the required properties of counterexamples to open non-existence conjectures for uniquely Hamiltonian graphs under various topological constraints. Concerning ourselves with a generalization of Sheehan's 1975 conjecture that no uniquely Hamiltonian graphs exist in the class of $\left(r \in 2 \mathbb{N}_{>1}\right.$ )-regular graphs (for $4 \leq r \leq 22$ ), Bondy \& Jackson's 1998 conjecture that no uniquely Hamiltonian graphs exist in the class of planar graphs having at most one vertex of degree $\leq 2$, and Fleischner's 2014 conjecture that no uniquely Hamiltonian graphs exist in the class of 4-vertex-connected graphs, we prove that each conjecture is false if and only if there exists a parsimonious reduction from \#SAT to counting Hamiltonian cycles on each graph class in question. As the existence of such a reduction allows for the encoding of arbitrary Unambiguous-SAT problem instances, by the Valiant-Vazirani theorem we have that hypothetical sets of counterexamples for each non-existence conjecture cannot belong to any graph class with a polynomial time testable property implying tractability for the Hamiltonian cycle decision problem (unless $N P=R P$ ).
\end{abstract}

Keywords: Hamiltonian cycle, uniquely Hamiltonian graph, Sheehan conjecture, Bondy \& Jackson conjecture, Fleischner conjecture, Valiant-Vazirani theorem, Unambiguous-SAT

\section{Introduction}

The question as to whether the members of a given graph class can be uniquely Hamiltonian (i.e., possess exactly one Hamiltonian cycle) has been the subject of considerable research in graph theory and related fields over the prior few decades. Interest in this question appears to have originated from a proof of C. A. B. Smith, reported by Tutte in 1946 [20], that the set of Hamiltonian cycles flowing through any edge of a cubic (i.e., 3-regular) graph must have even cardinality, and therefore, that any Hamiltonian cubic graph must have at least three Hamiltonian cycles. After a bit of an incubation period, in the 1970's Thomason [18] extended the result of C. A. B. Smith to all graphs whose vertices uniformly have odd degree, and Entringer and Swart [6] proved $\forall(n=2 k ; k \geq 11)$ that there exists a nearly cubic uniquely Hamiltonian graph on $n$ vertices with two vertices of degree 4 and with all remaining vertices of degree 3 (see "Theorem 5" of Ref. [6]). Briefly jumping ahead in time, we remark that in 2016 Gordon Royle [16] was able to establish that the smallest uniquely Hamiltonian graph of minimum degree 3 is of order 18 and nearly cubic, and that Goedgebeur et al. [11] shortly thereafter extended this result to prove the existence of nearly cubic uniquely Hamiltonian graphs on $n=2 k$ vertices $\forall k \geq 9$.

In the 1970's Sheehan also posed his famous conjecture [17] that no uniquely Hamiltonian 4-regular graphs can exist, which,

1 Graduate School of Arts and Sciences,

The University of Tokyo, Meguro, Tokyo 153-8902, Japan

a) rbarish@ims.u-tokyo.ac.jp

b) suyama@bio.c.u-tokyo.ac.jp in observation of the fact that every $\left(r \in 2 \mathbb{N}_{>0}\right)$-regular graph is the union of edge-disjoint spanning 2-factors, can be generalized to the statement that no $r$-regular uniquely Hamiltonian graphs exist $\forall\left(r \in 2 \mathbb{N}_{>1}\right)$. While there has been significant progress since this time on a proof of Sheehan's conjecture e.g., in 1998 Thomassen [19] proved the conjecture holds for $r \geq 300$, Ghandehari and Hatami subsequently reduced this bound to $r \geq 48$ (unpublished; personal communication cited in Ref. [12]), and most recently in 2006, Haxell et al. [12] proved that the conjecture holds true $\forall(r>22)$ - the conjecture remains open to this day. This is similarly the case for a still open conjecture of Bondy \& Jackson from 1998 [4], whereupon proving that any planar uniquely Hamiltonian graph must have at least two vertices of degree $\leq 3$, the authors put forth the supposition that no uniquely Hamiltonian planar graph exists having at most one vertex of degree $\leq 2$. Much more recently in 2014, after constructing an explicit and rather stunning example of a 3vertex-connected uniquely Hamiltonian graph having minimum degree 4 , and providing a method of constructing infinite families of such graphs, we additionally remark that Fleischner [8] posed the still open conjecture that no 4-vertex-connected uniquely Hamiltonian graph exists.

In this work, we examine the topological structure of sets of counterexamples, should they exist, to the open nonexistence conjectures of Sheehan [17], Bondy \& Jackson [4], and Fleischner [8]. In Theorem 1 through Theorem 4 we show that

This work was supported by a Grant-in-Aid for JSPS Research Fellow (18F18117 to R. D. Barish) from the Japan Society for the Promotion of Science. 
each of the aforementioned conjectures is false if and only if there exists a parsimonious reduction from \#SAT to the problem of counting Hamiltonian cycles on each of the relevant graph classes $-\left(r \in 2 \mathbb{N}_{>1}\right)$-regular graphs $(\forall r$ s.t. $4 \leq r \leq 22)$ in the case of Sheehan's generalized conjecture [17], planar graphs having at most one vertex of degree $\leq 2$ in the case of Bondy \& Jackson's conjecture [4], and 4-vertex-connected graphs in the case of Fleischner's conjecture [8]. To briefly clarify our meaning of the term parsimonious reduction, when reducing one integer function problem $f$ to another integer function problem $h$ via a many-one counting reduction (sometimes referred to as a "weakly parsimonious" reduction), one typically has two polynomial time compatible functions $R_{1}: \Sigma^{*} \longrightarrow \Sigma^{*}$ and $R_{2}: \mathbb{N} \longrightarrow \mathbb{N}$, such that $f(x)=R_{2}\left(h\left(R_{1}(x)\right)\right)$. If $R_{2}$ is the identity function we call the counting reduction a parsimonious reduction.

Here, the existence of a parsimonious reduction from \#SAT in each case allows us to efficiently encode arbitrary instances of a variant of satisfiability known as Unambiguous-SAT [22], where we are promised the existence of at most one satisfying assignment to a given Boolean formula. As a consequence, in Theorem 5 we are able to use the Valiant-Vazirani theorem, which establishes that no polynomial time algorithm for UnambiguousSAT can exist unless we have that $N P=R P$ (somewhat analogous to the notion that $P=N P$ ), to show no set of hypothetical counterexamples for any of the aforementioned non-existence conjectures can be contained (i.e., as a not necessarily proper subset) in any graph class with a polynomial time recognizable property implying an efficient solution for the Hamiltonian cycle decision problem. This subsequently allows us to exclude containment of hypothetical counterexample sets for each of the conjectures from at least 335 graph classes in the (July 7th, 2016) Information System on Graph Classes and their Inclusions (ISGCI) database [13] where we have that a cliquewidth bound both exists and can be detected in polynomial time.

\section{Complexity Theoretic Consequences for Counterexamples to the Three Nonexis- tence Conjectures}

Theorem 1. Sheehan's non-existence conjecture for uniquely Hamiltonian 4-regular graphs is false if and only if there exists a parsimonious reduction from \#SAT to the problem of counting Hamiltonian cycles on 4-regular graphs.

Proof. Our proof strategy will be to first show that the existence of a counterexample to Sheehan's non-existence conjecture for uniquely Hamiltonian 4-regular graphs [17] necessarily implies the existence of a special type of Exclusive-OR (XOR) gadget [10]. With this gadget in hand, we will then modify a reduction developed in 1976 by Garey et al. [10] — originally to reduce $S A T$ to the Hamiltonian cycle decision problem on cubic $\cap$ planar $\cap 3$-vertex-connected graphs - to achieve a parsimonious reduction from \#3SAT (for which there exists a parsimonious reduction from \#SAT) to the problem of counting Hamiltonian cycles on 4regular graphs.

To begin, we can understand Garey et al. Exclusive-OR (XOR) gadget [10] as a generalization of what we denote here as a special case of a quadrapole graph. We define this latter object, and a slight generalization of this object denoted a meta-quadrapole, as follows:

Definition 1: Quadrapole (variation on Kelmans [14] "fourpole"). Let $G$ be an arbitrary undirected simple graph with vertex set $V_{G}$ and edge set $E_{G}$, and let $Q$ be an induced subgraph of $G$ with vertex set $V_{Q} \subset V_{G}$ and edge set $E_{Q} \subset E_{G}$. We define $Q$ as a quadrapole if and only if there exists a set of exactly four edges $e_{1}, e_{2}, e_{3}, e_{4} \in E_{\text {cut }}$ where $E_{\text {cut }} \subset E_{G} \backslash E_{Q}$, each with one end at a (not necessarily distinct) vertex $v_{i} \in V_{\text {cut }}$ denoted a pole vertex, where $V_{\text {cut }} \subset V_{Q}$. With regard to a notion for quadrapole connectivity, let $Q^{*}$ correspond to a graph construction where we create a clique $C$ having the same cardinality as $V_{\text {cut }}$, and subsequently add an edge between each pole vertex $v_{i} \in V_{c u t}$ and a vertex in $C$ unique to $v_{i}$. Here, we call the quadrapole $Q \kappa$-vertexconnected (resp. $\kappa$-edge-connected) if and only if the vertex connectivity (resp. edge connectivity) of $Q^{*}$ is equal to $\kappa$.

Definition 2: Meta-quadrapole. A meta-quadrapole is a generalization of the definition of a quadrapole, wherein we allow pole vertices to correspond to a set of one or more vertices collectively referred to as a meta-vertex. Here, an edge with one end at a given meta-vertex of a meta-quadrapole, and one end outside the metaquadrapole at an arbitrary vertex $v_{q}$, implies the existence of a set of edges joining $v_{q}$ to all vertices composing the meta-vertex. With regard to a definition of $\kappa$-vertex-connectivity (resp. $\kappa$-edgeconnectivity) for meta-quadrapoles, we proceed exactly as in the case of a quadrapole, though here allowing all vertices composing each meta-vertex to correspond to pole vertices.

In Fig. 1 we illustrate all 9 possible manners in which a Hamiltonian cycle can traverse a quadrapole graph (i.e., perform an injective and surjective walk on the vertices of the quadrapole graph). However, note here that a meta-quadrapole may admit additional traversals types where, for instance, a Hamiltonian cycle ingresses and egresses once or multiple times via the same metavertex. We can now define an Exclusive-OR (XOR) gadget as a quadrapole or meta-quadrapole which strictly and only allows for a subset of the Fig. 1 Hamiltonian cycle traversals - specifically, either the "Pole 1" $\leftrightarrow$ "Pole 2" or "Pole 3" $\leftrightarrow$ "Pole 4" traversals: Definition 3: Exclusive-OR (XOR) gadget (based on the properties of a gadget originally described by Garey et al. [10]). Let $Q$ be a quadrapole with pole vertices "Pole 1", "Pole 2", "Pole 3", and "Pole 4", where any desired pole vertex "label" bijection is permissible and any two or more pole vertices may be equivalent. We call $Q$ an Exclusive-OR (XOR) gadget if and only if: (requirement 1 ) there exist $H_{1,2}>0$ manners in which a Hamiltonian cycle can traverse $Q$ (i.e., perform an injective and surjective walk on the vertices of $Q$ ) ingressing and egressing once via "Pole 1 " and "Pole 2"; (requirement 2) there exist $H_{3,4}>0$ manners in which a Hamiltonian cycle can traverse $Q$ ingressing and egressing once via "Pole 3" and "Pole 4"; (requirement 3) we have that $H_{1,2}=H_{3,4}$; (requirement 4 ) there exist no other possible types of Hamiltonian cycle traversals for $Q$.

To provide explicit examples to accompany Definition 3, in Fig. 2 (a.1) we show the original Garey et al. XOR gadget [10] used to reduce $S A T$ to the Hamiltonian cycle decision problem on cubic $\cap$ planar $\cap$ 3-vertex-connected graphs, in Fig. 2 (b.1) we show a variation on this XOR gadget used by Liśkiewicz et al. to 
HC Traversal
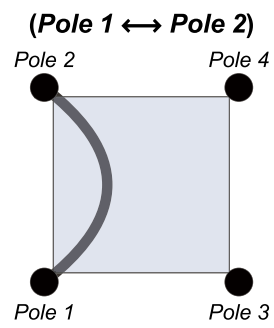

HC Traversal

(Pole $2 \longleftrightarrow$ Pole 3)

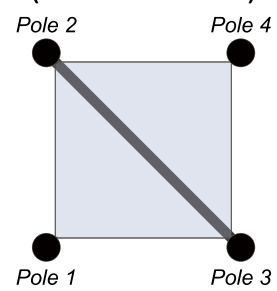

HC Traversal

(Pole $1 \longleftrightarrow$ Pole 2 \&

Pole $3 \longleftrightarrow$ Pole 4)

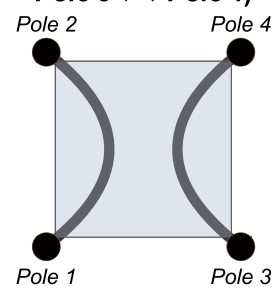

HC Traversal
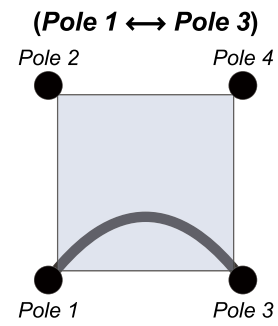

HC Traversal

(Pole $2 \longleftrightarrow$ Pole 4)

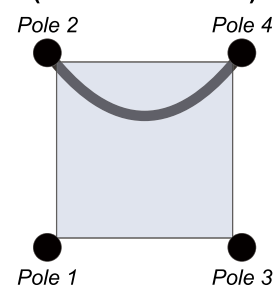

HC Traversal

(Pole $1 \longleftrightarrow$ Pole 3 \&

Pole $2 \longleftrightarrow$ Pole 4)

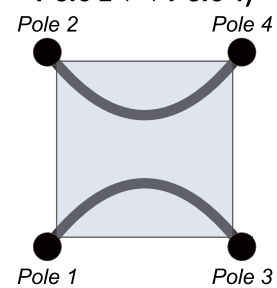

HC Traversal

(Pole $1 \longleftrightarrow$ Pole 4)

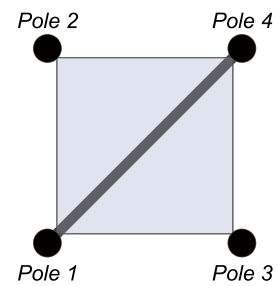

HC Traversal

(Pole $3 \longleftrightarrow$ Pole 4)

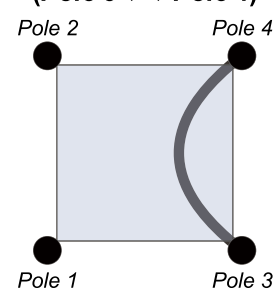

HC Traversal

(Pole $1 \longleftrightarrow$ Pole 4 \&

Pole $2 \longleftrightarrow$ Pole 3)

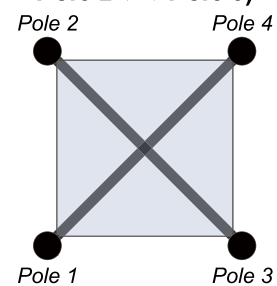

Fig. 1 Illustration of the 9 possible manners in which a Hamiltonian cycle may be permitted to traverse (i.e., perform an injective and surjective walk on the vertices of) a quadrapole graph.

reduce \#SAT to the problem of counting Hamiltonian cycles on cubic $\cap$ planar $\cap 2$-vertex-connected graphs, and in Fig. 2 (c.1) we show a novel XOR gadget. To see that all of the aforementioned XOR gadgets satisfy Definition 3, we can observe in the Fig. 2 (e) table - which lists the number of ways a Hamiltonian cycle can perform the traversals illustrated in Fig. 1 for each of the listed XOR gadgets - that Hamiltonian cycles can only ingress and egress these gadgets via a "Pole 1" $\leftrightarrow$ "Pole 2" or a "Pole 3" $\leftrightarrow$ "Pole 4" traversal. We can also observe in the Fig. 2 (e) table entry for the Fig. 2 (c.1) XOR gadget that it is possible for there to be exactly one manner in which a Hamiltonian cycle can flow between "Pole 1" $\leftrightarrow$ "Pole 2" or "Pole 3" $\leftrightarrow$ "Pole 4". To formalize this, we observe the following definition:

Definition 4: Unique Hamiltonian cycle traversal (unique HC traversal). A unique Hamiltonian cycle traversal (unique HC traversal) is an injective and surjective walk on the vertices of a subgraph or gadget - possibly ingressing or egressing the subgraph or gadget one or multiple times - which if permitted, will not destroy the property of the graph containing the subgraph or gadget from being uniquely Hamiltonian.

If we are to modify the Garey et al. [10] reduction to obtain a parsimonious reduction from \#3SAT to counting Hamiltonian cycles on 4-regular graphs, it is clear that we will require an XOR gadget corresponding to an induced subgraph of a 4-regular graph, where, like the Fig. 2 (c.1) XOR gadget, we additionally have that both the "Pole 1" $\leftrightarrow$ "Pole 2" or "Pole 3" $\leftrightarrow$ "Pole 4 " traversals are unique $\mathrm{HC}$ traversals. Accordingly, following the scheme illustrated in Fig. 3, we detail a method of transforming an arbitrary instance of a 4-regular uniquely Hamiltonian graph into an XOR gadget having these properties.

As shown in Fig. 3 (a), we begin by selecting an instance of a not-necessarily-induced $P_{3}$ subgraph where we have that exactly one edge of the subgraph (Bolded) is traversed by the unique Hamiltonian cycle in the graph, then delete both edges of the $P_{3}$ subgraph to generate quadrapole $(\alpha)$. We can observe that the unique $\mathrm{HC}$ traversals of quadrapole $(\alpha)$ correspond to a Hamiltonian cycle ingressing and egressing via "Pole 1" and "Pole 2" ("Pole 1" $\leftrightarrow$ "Pole 2"), or ingressing and egressing via "Pole 1" and "Pole 4" ("Pole 1" $\leftrightarrow$ "Pole 4"). Alternative, though not necessarily possible or unique $\mathrm{HC}$ traversals of quadrapole $(\alpha)$, correspond to a Hamiltonian cycle ingressing and egressing via "Pole 1" and "Pole 3" ("Pole 1" ↔ "Pole 3"), or a two-pass traversal where the Hamiltonian cycle ingresses and egresses via "Pole 1" and "Pole 3" and also ingresses and egresses via "Pole 2" and "Pole 4" ("Pole 1" $\leftrightarrow$ "Pole 3" \& "Pole 2" $\leftrightarrow$ "Pole 4").

Next, as illustrated in Fig. 3(b), we connect three copies of quadrapole $(\alpha)$, where (Bolded) edges in the assembly indicate all possible trajectories for ingressing and egressing Hamiltonian cycles, to generate quadrapole $(\beta)$ with a more restricted set of allowed Hamiltonian cycle traversals. Specifically, we observe that quadrapole $(\beta)$ can be traversed only by a Hamiltonian cycle ingressing and egressing via "Pole 1" and "Pole 2" ("Pole 1" $\leftrightarrow$ 
(a.1)

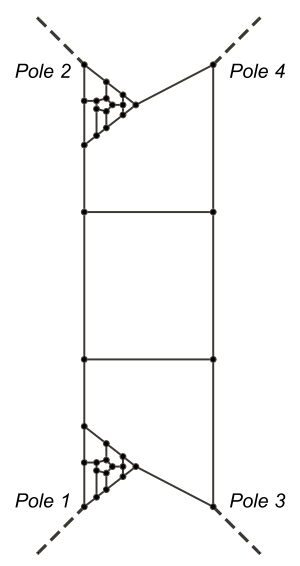

(b.1)

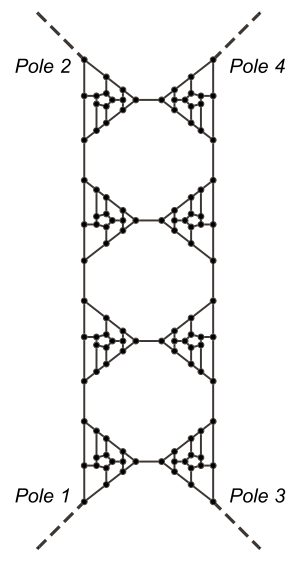

(c.1)

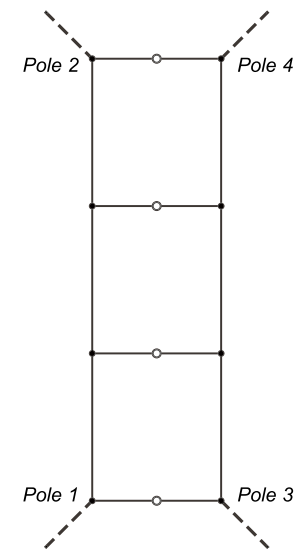

(a.2)

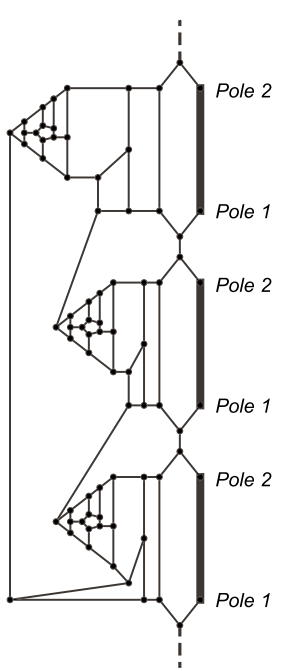

(b.2.1)

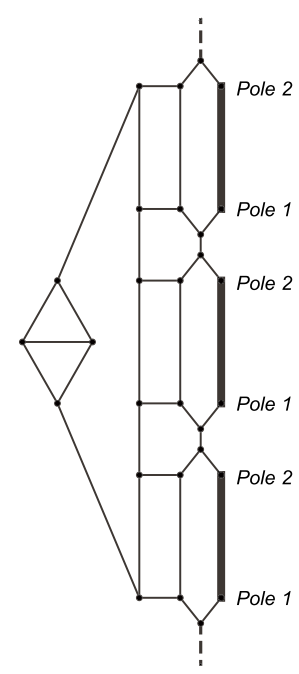

(c.2)

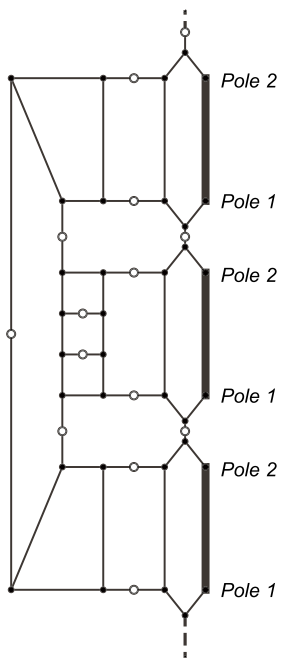

(d)

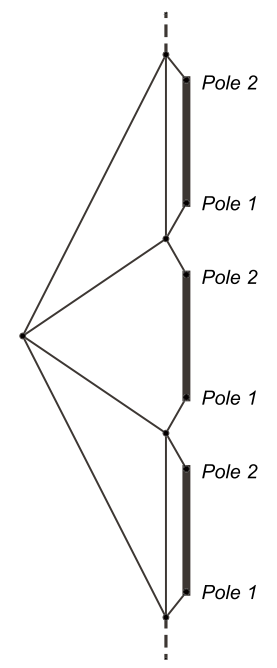

(e) HC Traversal Flow Counts for Quadrapole (XOR) Gadgets

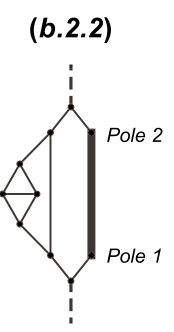

(a.1)
(b.1)

(b.1)
(c.1)

$\langle P 1, P 2\rangle\langle P 1, P 3\rangle\langle P 1, P 4\rangle\langle P 2, P 3$

\begin{tabular}{|c|c|c|c|c|c|c|c|c|}
\hline $2 x$ & o & 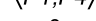 & 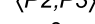 & $\langle F z, F 4\rangle$ & | ו & או & 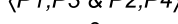 & $\langle P 1, P 4 \& P 2, P 3\rangle$ \\
\hline 8 & 0 & 0 & 0 & 0 & 8 & 0 & 0 & 0 \\
\hline 4096 & 0 & 0 & 0 & 0 & 4096 & 0 & 0 & 0 \\
\hline 1 & 0 & 0 & 0 & 0 & 1 & 0 & 0 & 0 \\
\hline
\end{tabular}

\section{(f) HC Traversal Flow Counts for OR Gadgets}

(a.2)
(b.2.1)
(b.2.2)
(c.2)
(d)

$\left.\begin{array}{cccccccccccc}\langle\text { bottom, middle,top }\rangle=\langle F, F, F\rangle & \langle T, F, F\rangle & \langle F, T, F\rangle & \langle F, F, T\rangle & \langle T, T, F\rangle & \langle T, F, T\rangle & \langle F, T, T\rangle & \langle T, T, T\rangle & \langle F\rangle & \langle T\rangle \\ & 0 & 216 & 216 & 216 & 288 & 288 & 288 & 288 & \text { N/A } & \text { N/A } \\ 0 & 2 & 2 & 2 & 4 & 4 & 4 & 6 & \text { N/A } & \text { N/A } \\ \text { N/A } & \text { N/A } & \text { N/A } & \text { N/A } & \text { N/A } & \text { N/A } & \text { N/A } & \text { N/A } & 0 & 2 \\ 0 & 1 & 1 & 1 & 1 & 2 & 1 & 1 & \text { N/A N/A } \\ & 0 & 1 & 1 & 1 & 1 & 2 & 1 & 1 & \text { N/A N/A }\end{array}\right)$

Fig. 2 Variants of XOR and OR gadgets compatible with the Garey et al. [10] reduction: (a.1) \& (a.2) the original XOR and 3-literal OR gadgets used by Garey et al. [10]; (b.1), (b.2.1), \& (b.2.2) XOR, 3-literal OR, and 1-literal OR gadgets used by Liśkiewicz et al. [15]; (c.1) \& (c.2) novel XOR and 3-literal OR gadget variants allowing for a parsimonious reduction from \#SAT to the problem of counting Hamiltonian cycles on planar $\cap$ subcubic graphs where we have that every vertex of degree 3 is adjacent to a single vertex of degree 2 (white colored vertices) and no two degree 2 vertices are adjacent; (d) novel 3-literal OR gadget used in parsimoniously reducing \#SAT to the problem of counting Hamiltonian cycles on graphs which are 4-regular and/or 4-vertex-connected. Table (e) lists "HC Traversal Flow Counts" for XOR gadgets, corresponding to the number of ways a Hamiltonian cycle can traverse each of the listed XOR gadgets by ingressing and egressing each gadget once or twice via the vertices labeled "Pole 1" (abbreviated as "P1"), "Pole 2" ("P2"), "Pole 3" ("P3"), and "Pole 4" ("P4"). Table (f) lists "HC Traversal Flow Counts" for OR gadgets, corresponding to the number of ways a Hamiltonian cycle can traverse each OR gadget for each possible truth assignment to the gadget's encoded literals. 
Not necessarily induced $P_{3}$ graph; edges deleted to create quadrapole $(\alpha)$

(a)

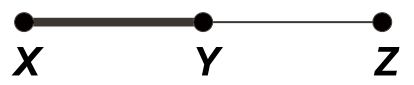

(b)

(c)

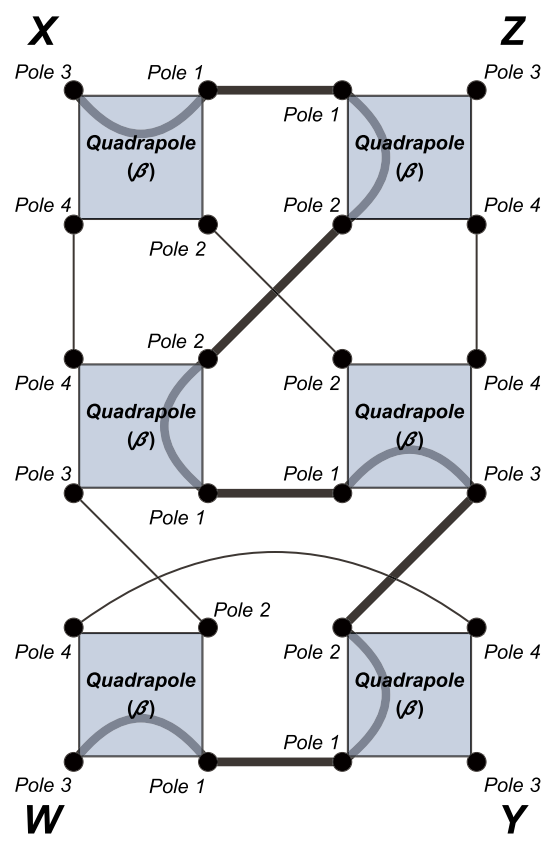

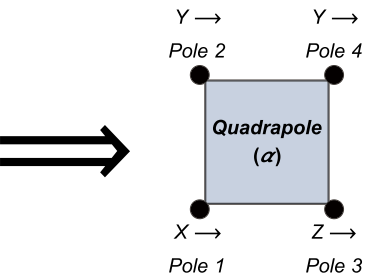

Quadrapole $(\alpha)$ Unique HC Traversals :: $\{($ Pole $1 \leftrightarrow$ Pole 2), (Pole $1 \leftrightarrow$ Pole 4) $\}$ Possible Alt. HC Traversals ::

$\{($ Pole $1 \leftrightarrow$ Pole 3),

(Pole $1 \leftrightarrow$ Pole $3 \&$ Pole $2 \leftrightarrow$ Pole 4 ) $\}$

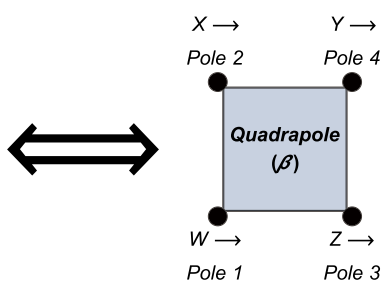

Quadrapole $(\beta)$ Unique HC Traversals :: $\{($ Pole $1 \leftrightarrow$ Pole 2$),($ Pole $1 \leftrightarrow$ Pole 3$)\}$ Possible Alt. HC Traversals :: None

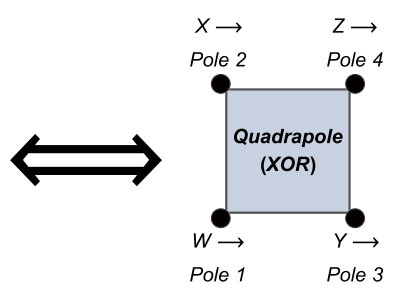

Quadrapole (XOR) Unique HC Traversals :: $\{($ Pole $1 \longleftrightarrow$ Pole 2$)$, (Pole $3 \longleftrightarrow$ Pole 4) $\}$

Possible Alt. HC Traversals :: None

Fig. 3 Construction used in the proof argument for Theorem 1 to transform an arbitrary instance of a 4-regular uniquely Hamiltonian graph into an XOR gadget that can be used to parsimoniously reduce \#SAT to the problem of counting Hamiltonian cycles on 4-regular graphs. In step (a) we select an instance of a not-necessarily-induced $P_{3}$ subgraph of a 4-regular uniquely Hamiltonian graph where we have that exactly one edge of the subgraph (Bolded) is traversed by the unique Hamiltonian cycle in the graph, then delete both edges of the $P_{3}$ subgraph to generate quadrapole $(\alpha)$ allowing for the listed types of Hamiltonian cycle traversals (i.e., injective and surjective walks on the quadrapole's vertices). In step (b) we connect three copies of quadrapole $(\alpha)$ as shown, where (Bolded) edges in the assembly indicate all possible trajectories for ingressing and egressing Hamiltonian cycles, to generate quadrapole $(\beta)$ with a more restricted set of allowed Hamiltonian cycle traversals. Finally, in step (c) we connect six copies of quadrapole $(\beta)$ together to form the desired XOR gadget, where (Bolded) edges in the assembly indicate one of the two possible (reflectionally symmetric) traversals for ingressing and egressing Hamiltonian cycles. 
"Pole 2"), or ingressing and egressing via "Pole 1" and "Pole 3" ("Pole 1" $\leftrightarrow$ "Pole 3"), that no alternate manners of traversal are possible, and that both traversal types are unique $\mathrm{HC}$ traversals.

For the last step, as illustrated in Fig. 3(c), we connect six copies of quadrapole $(\beta)$ together to form the desired XOR gadget, where (Bolded) edges in the assembly indicate one of the two possible (reflectionally symmetric) traversals for ingressing and egressing Hamiltonian cycles. We observe that this XOR gadget can be traversed by a Hamiltonian cycle ingressing and egressing via "Pole 1" and "Pole 2" ("Pole 1" $\leftrightarrow$ "Pole 2"), or by a Hamiltonian cycle ingressing and egressing via "Pole 3 " and "Pole 4" ("Pole 3" $\leftrightarrow$ "Pole 4"), that no alternate manners of traversal are possible, and that both traversal types are unique $\mathrm{HC}$ traversals.

We will now address how to use the XOR gadget we have just created to modify the Garey et al. reduction [10] to achieve a parsimonious reduction from \#3SAT to the problem of counting Hamiltonian cycles on 4-regular graphs. Here, let $R_{G J T}$ be an instance of a graph generated by the Garey et al. reduction, and let $R_{G J T}^{\prime}$ be an instance of $R_{G J T}$ where we make the following four modifications:

- (m1.1) we substitute the original Fig. 2 (a.1) and Fig. 2 (a.2) XOR and 3-literal OR gadgets with the novel XOR gadget we have just constructed (i.e., via the scheme illustrated in Fig. 3) and the novel Fig. 2 (d) 3-input OR gadget;

- (m1.2) in lieu of using a 2-input OR gadget as described on "pg. 710" of Garey et al. [10] — the purpose of this gadget originally being to ensure 3-vertex-connectivity of the graph generated by the reduction, which we remark can instead by ensured by "padding" any instance of a $3 S A T$ formula being reduced with a tautological clause - we simply use two edges to connect vertices $\left\{v_{11}, w_{11}\right\}$ and $\left\{v_{n 4}, w_{m 6}\right\}$, respectively (see "pg. 709" of Garey et al. [10] for an elaboration on the nature of these vertices);

- (m1.3) we do not perform the XOR "line crossing" planarization surgery detailed in "Fig. 4" of Garey et al. [10];

- (m1.4) in reference to the "variable" gadget vertices $\left\{v_{i 1}, v_{i 2}, v_{i 3}, v_{i 4}\right\}$ for $1 \leq i \leq n$, initially joined by "skeletal" edges of $R_{G J T}$ as detailed on "pg. 708-709" of Garey et al. [10], we add the edges $v_{i 1} \leftrightarrow v_{i 2}$ and $v_{i 3} \leftrightarrow v_{i 4}$ for all $1 \leq i \leq n$.

We can observe the following three lemmas concerning $R_{G J T}^{\prime}$ :

Lemma 1. $R_{G J T}^{\prime}$ will be 4-regular graph.

Proof. Observe that modification (m1.1) of $R_{G J T}$ replaces all XOR and 3-literal OR gadgets with variants that correspond to induced subgraphs of 4-regular graphs. Observe that modifications (m.1) through (m.3) yield a graph where all vertices are of degree 3 or 4 , and where the vertices of degree 3 strictly and only correspond to "variable" gadget vertices $\left\{v_{i 1}, v_{i 2}, v_{i 3}, v_{i 4}\right\}$ for $1 \leq i \leq n$. Finally, observe that the introduction of the edges $v_{i 1} \leftrightarrow v_{i 2}$ and $v_{i 3} \leftrightarrow v_{i 4}$ for all $1 \leq i \leq n$, as per modification (m.4), will increase the degree of each "variable" gadget vertex by exactly one, making $R_{G J T}^{\prime}$ a 4-regular graph.

Lemma 2. For a given instance of $R_{G J T}^{\prime}$ encoding an instance $\phi$ of the 3SAT decision problem, there will be a distinguishable set of Hamiltonian cycles corresponding to each satisfying instance of $\phi$, and each Hamiltonian cycle in a fgiven set will ingress and egress each XOR and 3-input OR gadget in the same manner.

Proof. This lemma follows directly from the description of the reduction given in Garey et al. [10]. Each 3-input OR gadget in the Garey et al. reduction corresponds to a specific 3-literal clause in the instance $\phi$ of the $3 S A T$, and the manner in which a Hamiltonian cycle ingress and egresses the gadget corresponds to a specific assignment of "True" and "False" values to the clause's literals. Furthermore, a specific set of traversals of each 3-input OR gadget in $R_{G J T}^{\prime}$ correspondingly forces a specific set of traversals of each XOR gadget in $R_{G J T}^{\prime}$.

To elaborate, observe either the Fig.2(a.2) embedding of the original Garey et al. 3-input OR gadget, or the Fig. 2 (b.2.1) embedding of the 3-input OR construct created by Liśkiewicz et al. [15] to obtain a many-one counting ("weakly parsimonious") reduction from \#SAT to the problem of counting Hamiltonian cycles on cubic $\cap$ planar $\cap 2$-vertex-connected graphs. During the reduction each of the three (thick black) edges on the right-handside of these 3-input OR gadgets are deleted and the resulting degree 1 vertices are identified as indicated with "Pole 1 " and "Pole 2" of a distinct instance of an appropriate XOR gadget. Here, each pair of edges connecting the 3-input OR to an instance of an XOR gadget corresponds to a clause literal.

We now remark that the global topology of the graph produced by the reduction, in this case $R_{G J T}^{\prime}$, will act to force all Hamiltonian cycles to ingress and egress a given 3-input OR gadget via the (thin dashed) edges at the top and bottom of the gadget (with respect to the illustrated embedding), and if the Hamiltonian cycle does not traverse (resp. traverses) a pair of edges connecting the 3-input OR gadget to an XOR we call the corresponding literal "True" (resp. "False"). Observing this correspondence between Hamiltonian cycle traversals of 3-input OR gadgets and literal truth assignments, in the Fig. 2 (f) table we provide counts for the number of ways a Hamiltonian cycle can perform each type of traversal of the illustrated OR gadgets in Fig. 2 (including the Fig. 2(b.2.2) illustration of a 1-input OR gadget created by Liśkiewicz et al. [15]). Here, to provide a specific example, for the Fig. 2 (a.2) 3-input OR gadget we have that there are exactly 288 manners in which a Hamiltonian cycle can traverse this OR gadget in a manner that sets the literals corresponding to the bottom, middle, and top pairs of edges to distinct XOR gadgets (with respect to the illustrated embedding) to "True", "False", and "True", respectively.

A specific set of traversals of the 3-input OR gadgets in $R_{G J T}^{\prime}$, corresponding to a specific truth assignment for the literals being encoded by each gadget, will in turn force Hamiltonian cycle flows through the "variable" gadgets of $R_{G J T}^{\prime}$ - pairs of loops connected by XOR gadgets shown, for example, along the righthand-side of the "Fig. 7" example construct in Garey et al.'s paper [10] — encoding truth assignments to variables that are consistent with the truth assignments to the aforementioned literals. It can accordingly be observed that a specific set of traversals of each 3-input OR gadget in $R_{G J T}^{\prime}$ forces a specific set of traversals of each XOR gadget in $R_{G J T}^{\prime}$.

Putting everything together, we have that there will be a set of Hamiltonian cycles corresponding to each satisfying instance of 
$\phi$ distinguishable by their manner of traversal of each 3-input OR gadget in $R_{G J T}^{\prime}$, and furthermore, that each Hamiltonian cycle in a given set will traverse each XOR gadget in $R_{G J T}^{\prime}$ in the same manner. This establishes the lemma.

Lemma 3. For a given instance of $R_{G J T}^{\prime}$ encoding an instance $\phi$ of the 3SAT decision problem, if all possible XOR and 3-input OR gadget Hamiltonian cycle traversals are unique HC traversals, then there will be exactly one Hamiltonian cycle in $R_{G J T}^{\prime}$ per satisfying instance of $\phi$.

Proof. From Lemma 2 we have that there will be a distinguishable set of Hamiltonian cycles in $R_{G J T}^{\prime}$ corresponding to each satisfying instance of $\phi$, and that each Hamiltonian cycle in a given set will ingress and egress each XOR and 3-input OR gadget in the same manner. By a careful examination of either $R_{G J T}$ or $R_{G J T}^{\prime}$, it can furthermore be observed that forcing a specific set of traversals of each XOR and 3-input OR gadget will correspondingly force the specific set of edges used to traverse vertices external to either type of gadget. Accordingly, the set of Hamiltonian cycles in $R_{G J T}^{\prime}$ corresponding to each satisfying instance of $\phi$ can be computed by simply multiplying together the number of ways in which a Hamiltonian cycle can perform each specified traversal of an XOR and 3-input OR gadget. Therefore, if all such gadget traversals are unique $\mathrm{HC}$ traversals, then there will be exactly one Hamiltonian cycle in $R_{G J T}^{\prime}$ per satisfying instance of $\phi$.

As we earlier established that the XOR gadget from modification (m1.1) used to construct $R_{G J T}^{\prime}$ allows for only unique HC traversals, it remains to show that the same is true of the Fig. 2 (d) 3-literal OR gadget exchanged in place of the Fig. 2 (a.2) Garey et al. 3-literal OR gadget. Here, observing the Fig. 2 (f) table we can see that, with the exception of exactly one case where exactly two literals are "True", the Fig. 2 (d) 3-literal OR gadget permits only unique $\mathrm{HC}$ traversals. As we can parsimoniously reduce \#3SAT to variants where this pathological permutation of truth assignments in a clause never occurs (see e.g., Ref. [21]), this establishes the lemma.

To finish the proof of the theorem at hand, observe by Lemma 1 that the modified graph $R_{G J T}^{\prime}$ will be 4-regular, and by Lemma 3 that there will be exactly one Hamiltonian cycle in $R_{G J T}^{\prime}$ per satisfying assignment for the encoded instance of \#3SAT. Accordingly, we have a method of using an arbitrary counterexample to Sheehan's nonexistence conjecture [17] for 4-regular uniquely Hamiltonian graphs to reduce \#3SAT to the problem of counting Hamiltonian cycles on this class of graphs.

Theorem 2. Sheehan's generalized non-existence conjecture for uniquely Hamiltonian $\left(r \in 2 \mathbb{N}_{>1}\right.$ )-regular graphs $(\forall r$ s.t. $4 \leq$ $r \leq 22$ ) is false if and only if there exists a parsimonious reduction from \#SAT to the problem of counting Hamiltonian cycles on $\left(r \in 2 \mathbb{N}_{>1}\right.$ )-regular graphs.

Proof. Let $R_{G J T}$ be an instance of a graph generated by the Garey et al. reduction [10] (as in the Theorem 1 proof argument), and let $\widehat{R_{G J T}}$ be an instance of this graph where we make the following four modifications:

- (m2.1) we substitute the original Fig. 2 (a.1) and Fig. 2 (a.2) XOR and 3-literal OR gadgets with the novel XOR and 3-literal OR gadgets in Fig. 2(c.1) and Fig. 2(c.2), respectively;
- $(\mathrm{m} 2.2)$ we perform a procedure identical to $(\mathrm{m} 1.2)$ in the Theorem 1 proof argument with the added step that we subsequently subdivide the edge $v_{11} \leftrightarrow w_{11}$;

- (m2.3) concerning the XOR "line crossing" planarization scheme illustrated in "Fig. 4" of Garey et al. [10], we everywhere perform the variation of this surgery illustrated in Fig. 5 - where XOR "lines" correspond to the Fig. 2 (c.1) XOR in the manner shown in Fig.5(a), we perform the surgery to resolve all pairs of XOR "line crossings" (an example of which is shown in Fig. 5 (b)), and an explicit example of the result of the surgery is illustrated in Fig. 5 (c);

- (m2.4) in reference to the "variable" gadget vertices $\left\{v_{i 1}, v_{i 2}, v_{i 3}, v_{i 4}\right\}$ for $1 \leq i \leq n$, initially joined by "skeletal" edges of $R_{G J T}$ as detailed on "pg. 708-709" of Garey et al. [10], we subdivide every edge $v_{i 2} \leftrightarrow v_{i 3}$ for all $1 \leq i \leq n$ and every edge $v_{i 4} \leftrightarrow v_{i+1}$ for all $1 \leq i \leq n-1$.

It is straightforward to observe that $\widehat{R_{G J T}}$ will be a subcubic graph having the special property that every vertex of degree 3 is adjacent to a single vertex of degree 2 and that no two vertices of degree 2 will be adjacent. Here, see for instance the positions of the (white colored) degree 2 vertices in the Fig. 2 (c.1) and Fig. 2 (c.2) illustration of the XOR and 3-input OR gadget variants used for modification $(\mathrm{m} 2.1)$, and observe that the variation of the XOR "line crossing" planarization surgery we employ as per modification (m2.3) (illustrated in Fig. 5) preserves the aforementioned properties. Accordingly, we can construct an $\left(r \in 2 \mathbb{N}_{>1}\right)$-regular quadrapole gadget simulating the traversal properties of a subdivided edge of a subcubic graph and substitute this gadget in place of every subdivided edge in $\widehat{R_{G J T}}$ to make the graph $\left(r \in 2 \mathbb{N}_{>1}\right)$-regular. Furthermore, if all possible traversals through this quadrapole are unique $\mathrm{HC}$ traversals, we can do so without introducing additional Hamiltonian cycles per satisfying instance of the encoded instance of \#SAT.

To construct such a quadrapole, we begin by following the procedure in the Theorem 1 proof argument, though in this context starting with an $\left(r \in 2 \mathbb{N}_{>1}\right)$-regular graph. This yields a quadrapole $(\alpha)$ having exactly the same types of unique HC traversals and alternative Hamiltonian cycle traversals as in the Theorem 1 reduction. As shown in Fig. 4(b), we then diverge from the Theorem 1 proof argument in using three copies of quadrapole $(\alpha)$ to create a quadrapole $(\beta)$ having the same traversal properties for Hamiltonian cycles as the illustrated subdivided edge of a subcubic graph. Specifically, we observe that quadrapole $(\beta)$ can be traversed by a Hamiltonian cycle ingressing and egressing via "Pole 1" and "Pole 2" ("Pole 1" $\leftrightarrow$ "Pole 2"), "Pole 1" and "Pole 4" ("Pole 1" $\leftrightarrow$ "Pole 4"), "Pole 2" and "Pole 3" ("Pole 2" $\leftrightarrow$ "Pole 3"), or "Pole 3" and "Pole 4" ("Pole 3 " $\leftrightarrow$ "Pole 4"), that no alternate manners of traversal are possible, and that all of these traversal types are unique HC traversals.

We can now substitute quadrapole $(\beta)$ in place of any subdivided edge. To do so, let $v_{a} \leftrightarrow v_{b} \leftrightarrow v_{c}$ be a subdivided edge of a subcubic graph $G$ where $v_{a}$ and $v_{b}$ have degree 3 and $v_{b}$ has degree 2 , let $e_{(a, 1)}$ and $e_{(a, 2)}$ be the two edges with one endpoint at $v_{a}$ and one endpoint at a vertex other than $v_{b}$, and let $e_{(c, 1)}$ and $e_{(c, 2)}$ be the two edges with one endpoint at $v_{c}$ and one endpoint at a vertex 


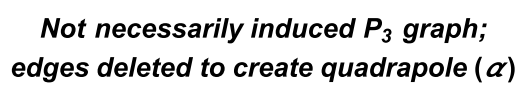

(a)

(b)

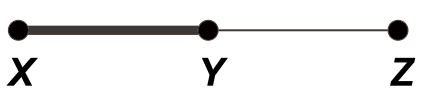

Z
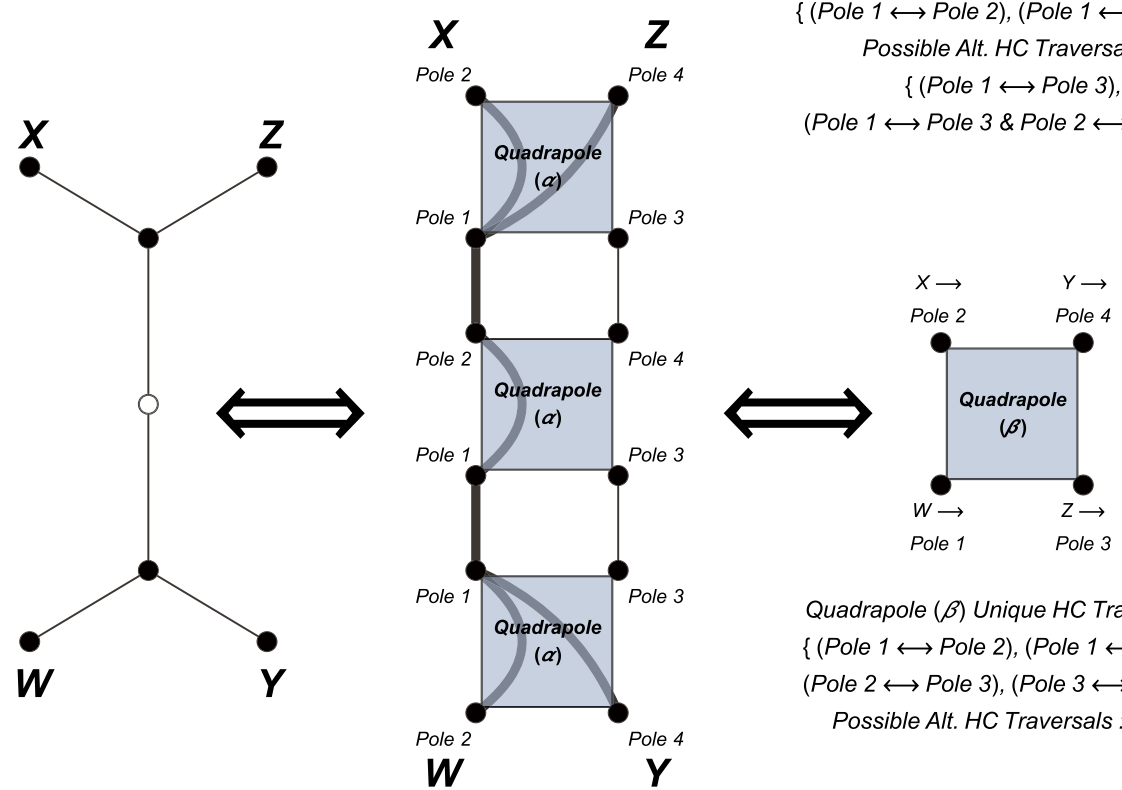

Quadrapole $(\alpha)$ Unique HC Traversals ::

$\{($ Pole $1 \leftrightarrow$ Pole 2$),($ Pole $1 \longleftrightarrow$ Pole 4$)\}$

Possible Alt. HC Traversals ::

$\{($ Pole $1 \longleftrightarrow$ Pole 3$)$,

(Pole $1 \longleftrightarrow$ Pole $3 \&$ Pole $2 \longleftrightarrow$ Pole 4$)\}$

Quadrapole $(\beta)$ Unique HC Traversals ::

$\{($ Pole $1 \longleftrightarrow$ Pole 2$),($ Pole $1 \longleftrightarrow$ Pole 4$)$

(Pole $2 \longleftrightarrow$ Pole 3), (Pole $3 \longleftrightarrow$ Pole 4) \}

Possible Alt. HC Traversals :: None

Fig. 4 Construction used in the proof argument for Theorem 2 to transform an arbitrary instance of an $r$-regular uniquely Hamiltonian graph into a graph gadget which can be used to simulate the Hamiltonian cycle traversal properties of a subdivided edge of a subcubic graph. In step (a) we select an instance of a not-necessarily-induced $P_{3}$ subgraph of an $\left(r \in 2 \mathbb{N}_{>1}\right)$-regular uniquely Hamiltonian graph where we have that exactly one edge of the subgraph (Bolded) is traversed by the unique Hamiltonian cycle in the graph, then delete both edges of the $P_{3}$ subgraph to generate quadrapole $(\alpha)$ allowing for the listed types of Hamiltonian cycle traversals. In step (b) we connect three copies of quadrapole $(\alpha)$ as shown, where (Bolded) edges in the assembly indicate all possible trajectories for ingressing and egressing Hamiltonian cycles, to generate a quadrapole $(\beta)$ having the same traversal properties for Hamiltonian cycles as the illustrated subdivided edge of a subcubic graph.

other than $v_{b}$. Here, when we substitute an instance of quadrapole $(\beta)$ in place of this subdivided edge, we delete $v_{a}, v_{b}$, and $v_{c}$, reconnect $e_{(a, 1)}$ and $e_{(a, 2)}$ to "Pole 1 " and "Pole 3 " of quadrapole $(\beta)$ in any order, and reconnect $e_{(c, 1)}$ and $e_{(c, 2)}$ to "Pole 2 " and "Pole 4" of quadrapole $(\beta)$ in any order. Performing this procedure at every subdivided edge of $G$ will yield an $\left(r \in 2 \mathbb{N}_{>1}\right)$-regular graph having the same Hamiltonian cycle count as the original subcubic graph.

Putting everything together, we have that a uniquely Hamiltonian $\left(r \in \mathbb{N}_{>1}\right.$ )-regular graph implies the existence of a parsimonious reduction from \#SAT to the problem of counting Hamiltonian cycles on the same class of $r$-regular graphs.

Theorem 3. Bondy $\mathcal{F}$ Jackson's non-existence conjecture for uniquely Hamiltonian planar graphs having at most one vertex of degree $\leq 2$ is false if and only if there exists a parsimonious reduction from \#SAT to the problem of counting Hamiltonian cycles on planar graphs having at most one vertex of degree $\leq 2$.

Proof. We begin by observing that Bondy \& Jackson's non-existence conjecture for uniquely Hamiltonian planar graphs having at most one vertex of degree $\leq 2$ is false if and only if the conjecture that the class of planar $\cap$ minimum degree 3 graphs contains no uniquely Hamiltonian graphs is false (see "Theorem 3.5" of Ref. [11]). The forward direction is a straightforward consequence of the fact that the class of planar $\cap$ minimum degree 3 graphs is a strict subset of the class of planar graphs having at most one vertex of degree $\leq 2$. Furthermore, as noted by Goedgebeur et al. [11], we can subdivide any edge traversed by the Hamiltonian cycle in the uniquely Hamiltonian planar $\cap$ minimum degree 3 graph to create a uniquely Hamiltonian planar graph having exactly one vertex of degree 2 . In the other direction, let $G_{1}$ and $G_{2}$ be two isomorphic copies of a counterexample to Bondy \& Jackson's non-existence conjecture, each having a single vertex of degree 2 . Let $v_{a}$ and $w_{a}$ be the vertices of degree 2 in $G_{1}$ and $G_{2}$, respectively, let $v_{a}$ be adjacent to a pair of vertices $\left\{v_{b}, v_{c}\right\}$ of degree $\geq 3$, and let $w_{a}$ be adjacent to a pair of vertices $\left\{w_{b}, w_{c}\right\}$ of degree $\geq 3$. Here, we can construct a uniquely Hamiltonian planar $\cap$ minimum degree 3 graph by deleting $v_{a}$ and $w_{a}$, then adding edges between the pairs of vertices $\left\{v_{b}, w_{b}\right\}$ and $\left\{v_{c}, w_{c}\right\}$. 

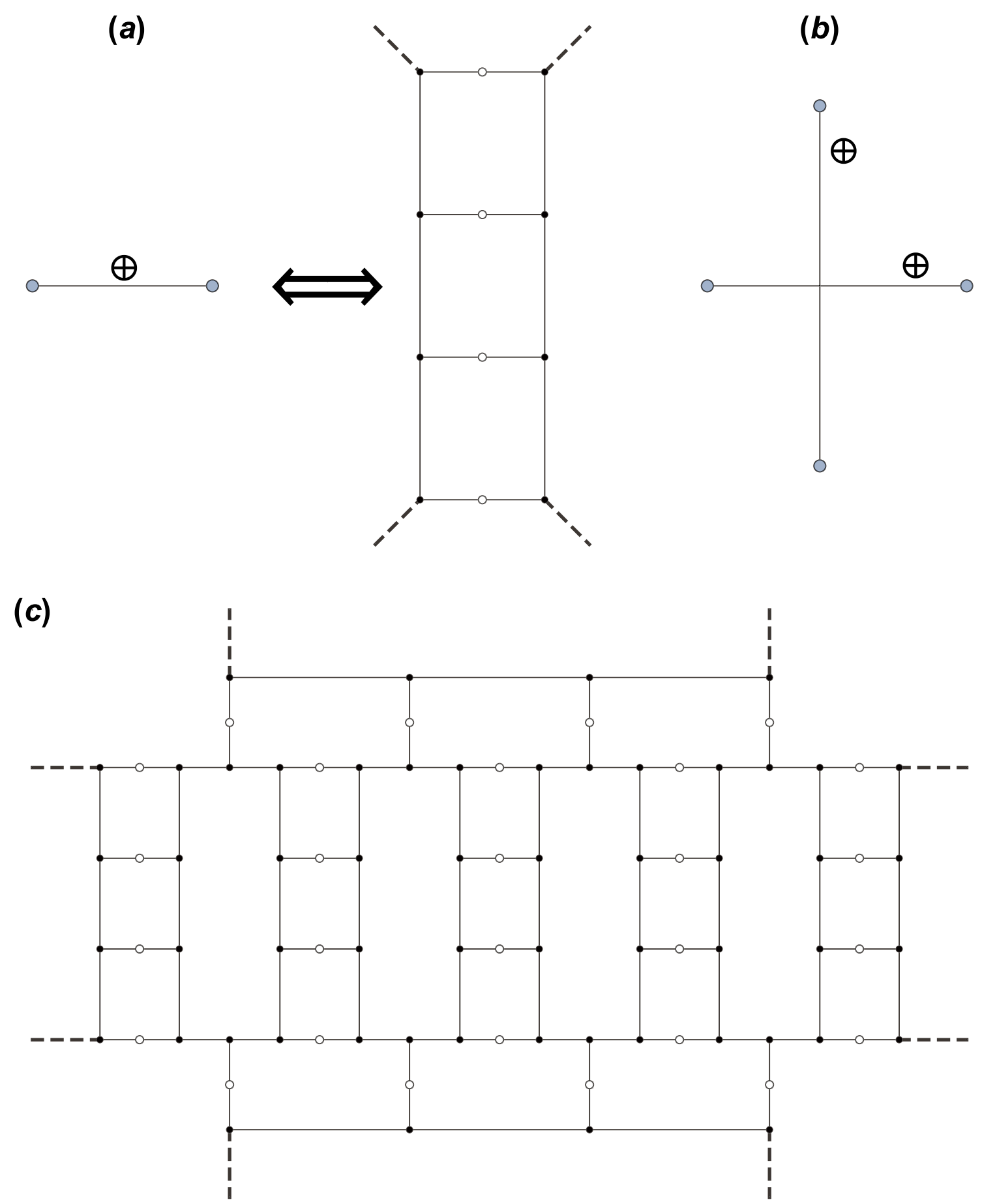

Fig. 5 Variation on the XOR "line crossing" planarization scheme from "Fig. 4" of Garey et al. [10], used in the proof argument for Theorem 2. (a) illustration of how an XOR gadget (the Fig. 2(c.1) XOR is shown) can be abstracted as an "XOR line"; (b) illustration of an XOR "line crossing"; (c) scheme for planarizing XOR "line crossings" using the Fig. 2 (c.1) XOR gadget which preserves the property of each degree 3 vertex being adjacent to exactly one degree 2 vertex (white colored vertices) and no two degree 2 vertices being adjacent.

Putting everything together, it now suffices to show that Bondy \& Jackson's non-existence conjecture is false if and only if there exists a parsimonious reduction from \#SAT to the problem of counting Hamiltonian cycles on planar $\cap$ minimum degree 3 graphs. To do so, we can simply observe that any planar $\cap$ minimum degree 3 uniquely Hamiltonian graph must possess a not-necessarily-induced $P_{3}$ subgraph, that the Fig. 2 (d) 3-input OR gadget can exist as an induced subgraph of a planar $\cap$ mini- mum degree 3 graph, and that none of the procedures performed in the Theorem 1 reduction prevent the final graph generated by the reduction from being a planar $\cap$ minimum degree 3 graph. Accordingly, we can follow along exactly the lines of the Theorem 1 proof argument - though in this context using a planar $\cap$ minimum degree 3 uniquely Hamiltonian graph to construct the XOR gadget - to parsimoniously reduce \#SAT to the problem of counting Hamiltonian cycles on planar $\cap$ minimum degree 3 
graphs, yielding the theorem.

Theorem 4. Fleischner's non-existence conjecture for 4-vertexconnected uniquely Hamiltonian graphs is false if and only if there exists a parsimonious reduction from \#SAT to the problem of counting Hamiltonian cycles on 4-vertex-connected graphs.

Proof. We can observe that the XOR gadget constructions used in the proof arguments of Theorem 1 and Theorem 2 (illustrated in Fig. 3 and Fig. 4, respectively) fail to preserve 4-vertexconnectivity for the trivial reason that quadrapole $(\alpha)$ from both Fig. 3 (a) and Fig. 4 (a) only has three distinct pole vertices (i.e., one pole vertex is joined to two outgoing edges). As a consequence, any graph having this quadrapole as an induced subgraph will be at most 3-vertex-connected.

We instead proceed to build an XOR gadget via the construction shown in Fig. 6. Let $G$ be an arbitrary instance of a 4-vertexconnected uniquely Hamiltonian graph with vertex set $V_{G}$. We first create quadrapole $(\alpha)$ by deleting an arbitrary vertex $v_{q} \in V_{G}$, then denoting the vertices formerly adjacent to $v_{q}$ as quadrapole $(\alpha)$ pole vertices in the manner illustrated in Fig. 6 (a). To address the case where the degree of $v_{q}$ is $\geq 5$, we allow quadrapole $(\alpha)$ to be a meta-quadrapole where the (white) colored pole vertex (i.e., "Pole 4") corresponds to a set of vertices of arbitrary cardinality, though under the constraint that none of the vertices in this set are allowed to be adjacent to either of the two edges originally used by the unique Hamiltonian cycle to ingress and egress the vertex $v_{q}$. We observe here that as a consequence of the allowed traversals for the vertex $v_{q}$ in the uniquely Hamiltonian graph $G$, the (meta-)quadrapole ( $\alpha$ ) only allows for the traversal types listed in Fig. 6(a). In particular, this consists of a single type of unique $\mathrm{HC}$ traversal where the (meta-)quadrapole is ingressed and egressed once via "Pole 1" and "Pole 2" ("Pole 1" $\leftrightarrow$ "Pole 2"), and all possible types of traversals, which may or may not be possible and which may or may not be unique HC traversals, where a Hamiltonian cycle ingresses and egresses the (meta-)quadrapole more than once (e.g., via pairs of vertices corresponding to a Pole 4 meta-vertex).

As illustrated in Fig. 6(b), we next create a new quadrapole $(\beta)$ where only a unique $\mathrm{HC}$ traversal via "Pole 1" and "Pole 2" ("Pole 1" $\leftrightarrow$ "Pole 2") is permitted. Here, simply observe that none of the copies of quadrapole $(\alpha)$ can be ingressed and egressed by a Hamiltonian cycle more than once in the Fig. 6 (b) construction. Subsequently, as illustrated in Fig. 6(c), we create an XOR gadget which will be 4-vertex-connected if and only if $G$ is 4 -vertex-connected. As in the Theorem 1 proof argument, we observe that this XOR gadget can be traversed by a Hamiltonian cycle ingressing and egressing via "Pole 1" and "Pole 2" ("Pole 1" ↔ "Pole 2"), or by a Hamiltonian cycle ingressing and egressing via "Pole 3" and "Pole 4" ("Pole 3" $\leftrightarrow$ "Pole 4"), that no alternate manners of traversal are possible, and that both traversal types are unique HC traversals.

We can now observe that the XOR gadget we have just constructed is a 4-vertex-connected quadrapole, and furthermore, that the Fig. 2(d) 3-input OR gadget can exist as an induced subgraph of a 4-vertex-connected graph. Here, letting $R_{G J T}^{\prime}$ be an instance of graph produced by the modification of the Garey et al. reduction [10] detailed in Theorem 1, and letting
$R_{G J T}^{\prime \prime}$ be a variation of $R_{G J T}^{\prime}$ where we use the 4-vertex-connected quadrapole we have just constructed instead of the XOR from the proof argument for Theorem 1, we can observe the following lemma:

Lemma 4. $R_{G J T}^{\prime \prime}$ is 4-vertex-connected.

Proof. "On "pg. 711" of Garey et al. [10], the authors write the following concerning their reduction:" "... We leave to the reader the straightforward but tedious verification that the graph is cubic and triply connected. Basically, all one need do is verify that our special subgraphs have these properties (or would if their external edges were connected by external paths) and that the overall superstructure does also... ". Performing this analysis, it is likewise straightforward to determine that any graph produced by the Garey et al. reduction will be essentially-4-vertexconnected - meaning that the cardinality of the minimum vertex cut whose removal decomposes the graph into two connected components, each consisting of at least two vertices, is of size at least 4 - if we substitute the original Fig. 2 (a.1) and Fig. 2 (a.2) XOR and 3-literal OR gadgets with the novel XOR gadget we have just constructed via the scheme illustrated in Fig. 6 and the novel Fig. 2(d) 3-input OR gadget. Now observe that the procedure to construct $R_{G J T}^{\prime}$ given in the Theorem 1 proof argument ensures that the graph has minimum degree 4 , and that our modification of $R_{G J T}^{\prime}$ to create $R_{G J T}^{\prime \prime}$ will not reduce the minimum vertex degree. Accordingly, we have that this procedure will eliminate all essential 3-vertex cuts, leaving $R_{G J T}^{\prime \prime} 4$-vertex-connected. $\quad \square$

Observing Lemma 4, we can now follow along exactly the lines of the Theorem 1 proof argument - though in this context using the XOR gadget given by the construction in Fig. 6 - to parsimoniously reduce \#SAT to the problem of counting Hamiltonian cycles on 4-vertex-connected graphs.

Corollary 1. If a uniquely Hamiltonian 4-regular $\cap$ 4-vertexconnected graph exists, then there exists a parsimonious reduction from \#SAT to the problem of counting Hamiltonian cycles on 4-regular $\cap 4$-vertex-connected graphs.

Proof. Observing Lemma 1, that the only difference between the reductions in the Theorem 1 and Theorem 4 proof arguments concerns the use of a distinct XOR gadget, and finally that the XOR constructed in the Theorem 4 proof argument will be 4-regular $\cap 4$-vertex-connected if the initial uniquely Hamiltonian graph is 4-regular $\cap 4$-vertex-connected, we have the corollary.

Theorem 5. Unless $N P=R P$, any set of counterexamples to the following non-existence conjectures cannot be contained in a graph class with a polynomial time recognizable property implying polynomial time tractability of the Hamiltonian cycle decision problem: (1) Sheehan's generalized non-existence conjecture [17] for uniquely Hamiltonian $\left(r \in 2 \mathbb{N}_{>1}\right)$-regular graphs ( $\forall r$ s.t. $4 \leq r \leq 22)$; (2) Bondy E Jackson's non-existence conjecture [4] for planar graphs having at most one vertex of degree $\leq$ 2; (3) Fleischner's non-existence conjecture [8] for 4-vertexconnected uniquely Hamiltonian graphs.

Proof. By the Valiant-Vazirani theorem [22] we have that no polynomial time procedure can exist for solving arbitrary instances of Unambiguous-SAT unless $N P=R P$, where Unambiguous-SAT is once again a variant of $S A T$ where we 


\section{(a)}

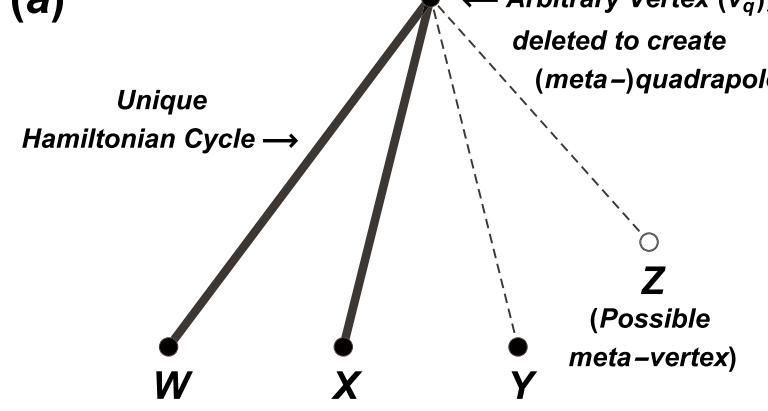

(b)
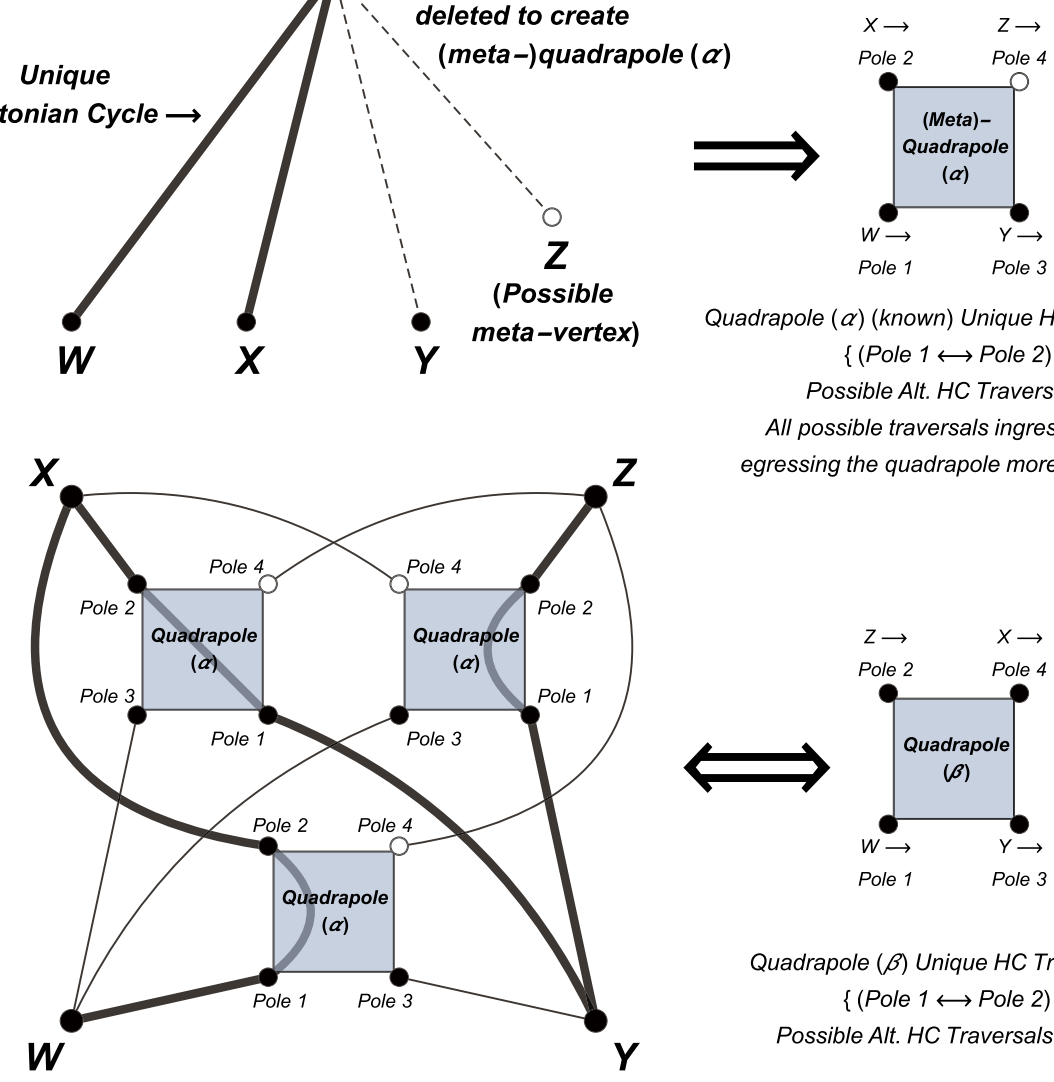

Quadrapole ( $\alpha$ ) (known) Unique HC Traversals ::

$\{($ Pole $1 \leftrightarrow$ Pole 2) $\}$

Possible Alt. HC Traversals ::

All possible traversals ingressing and

egressing the quadrapole more than once.

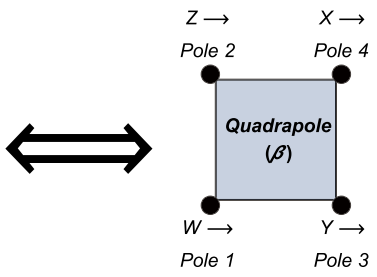

Quadrapole ( $\beta$ ) Unique HC Traversals :: $\{($ Pole $1 \leftrightarrow$ Pole 2$)\}$

Possible Alt. HC Traversals :: None

(c)
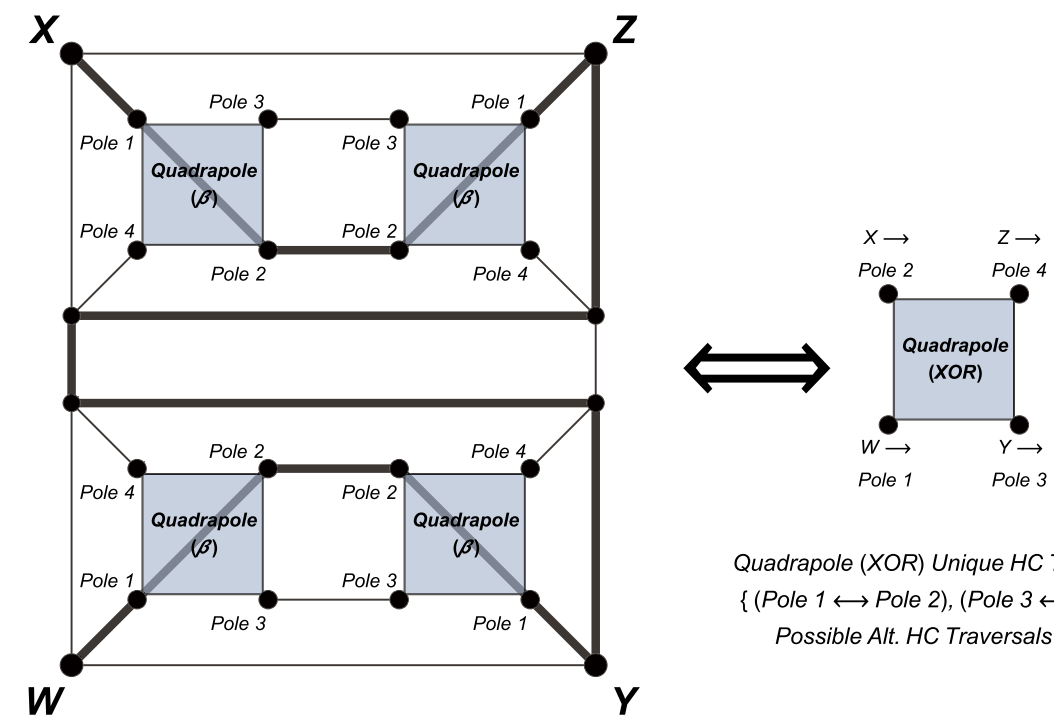

Quadrapole (XOR) Unique HC Traversals ::

$\{($ Pole $1 \longleftrightarrow$ Pole 2$),($ Pole $3 \longleftrightarrow$ Pole 4$)\}$

Possible Alt. HC Traversals :: None

Fig. 6 Construction used in the proof argument for Theorem 4 to transform an arbitrary instance of a 4-vertex-connected uniquely Hamiltonian graph into an XOR gadget. In step (a) we select an arbitrary vertex, $v_{q}$, of a 4-vertex-connected uniquely Hamiltonian graph, where we necessarily have that exactly two edges (Bolded) are traversed by the unique Hamiltonian cycle in the graph, and delete $v_{q}$ to generate quadrapole $(\alpha)$ allowing for the listed types of Hamiltonian cycle traversals. Here, to address the case where the degree of $v_{q}$ is $\geq 5$, we allow quadrapole $(\alpha)$ to be a metaquadrapole (see Definition 2) where the (white) colored pole vertex (i.e., "Pole 4") corresponds to a set of vertices of arbitrary cardinality. In step (b) we use four new vertices to wire together three copies of quadrapole $(\alpha)-$ (Bolded) edges in the assembly indicate all possible trajectories for ingressing and egressing Hamiltonian cycles — to generate quadrapole $(\beta)$ which allows for only one unique manner of Hamiltonian cycle traversal. Finally, in step (c) we use eight new vertices to assemble four copies of quadrapole $(\beta)$ together to form the desired XOR gadget, where (Bolded) edges in the assembly indicate one of the two possible (reflectionally symmetric) traversals for ingressing and egressing Hamiltonian cycles. 
are tasked with deciding the satisfiability of a Boolean formula with the promise that at most one solution can exist. Here, from the constructions in Theorem 1 through Theorem 4, we have a method of parsimoniously reducing \#SAT to the problem of counting Hamiltonian cycles on: $\left(r \in 2 \mathbb{N}_{>1}\right)$-regular graphs $(\forall r$ s.t. $4 \leq r \leq 22)$ in the case of Sheehan's generalized nonexistence conjecture [17] (Theorem 1 and Theorem 2); planar graphs having at most one vertex of degree $\leq 2$ in the case of Bondy \& Jackson's non-existence conjecture [4] (Theorem 3); and 4-vertex-connected graphs in the case of Fleischner's nonexistence conjecture [8] (Theorem 4). Accordingly, we have an explicit method of parsimoniously reducing Unambiguous-SAT to the problem of deciding the existence of a unique Hamiltonian cycle on each of these graph classes.

It is now straightforward to observe that a polynomial time algorithm for Unambiguous-SAT is implied by the combination of: (1) the existence of a set of one or more counterexamples to any of the aforementioned non-existence conjectures; and (2) the containment of this set in a graph class with an efficiently recognizable property implying polynomial time tractability of the Hamiltonian cycle decision problem. Consider that we can simply encode an arbitrary instance of Unambiguous-SAT in an instance of the Hamiltonian cycle decision problem on the relevant graph class and test for the property in question. If this property exists, we have a polynomial time algorithm for the Unambiguous-SAT instance, and if not, we can determine that the Unambiguous-SAT instance is unsatisfiable. As no polynomial time algorithm for Unambiguous-SAT can exist unless $N P=R P[22]$, the theorem follows.

\section{The Structure of Hypothetical Counterex- amples to Sheehan's, Bondy \& Jackson's, and Fleischner's Non-existence Conjectures}

By Theorem 5, we have that any set of counterexamples to Sheehan's [17], Bondy \& Jackson's [4], and Fleischner's [8] nonexistence conjectures for uniquely Hamiltonian graphs cannot be contained in any graph class having a polynomial time recognizable property implying an efficient algorithm for the Hamiltonian cycle decision problem (unless $N P=R P$ ). Observing the existence of cliquewidth parameterized "slice-wise polynomial" (XP) algorithms for the Hamiltonian cycle decision problem [7], [9] (which have time complexities of the form $O\left(n^{f(k)}\right)$ where $k$ is the cliquewidth), we are accordingly able to exclude containment of counterexamples for the aforementioned conjectures from any graph class having a polynomial time recognizable cliquewidth bound. By the following proposition, this includes at least 335 classes of bounded cliquewidth graphs in the (July 7th, 2016) version of the ISGCI database [13]:

Proposition 1. With the exception of the class of cliquewidth 4 graphs, there are known polynomial time algorithms for deciding if a graph has cliquewidth $\leq k$ on all 335 remaining graph classes listed as having bounded cliquewidth in the (July 7th, 2016) Information System on Graph Classes and their Inclusions (ISGCI) database [13].

Proof. Of the 336 graph classes in the (July 7th, 2016) ISGCI database [13] listed as having bounded cliquewidth, polynomial time recognition algorithms are either directly provided or cited for all but the following four classes of graphs: binary tree $\cap$ partial grid graphs, chordal $\cap$ hamiltonian $\cap$ planar graphs, thick tree graphs, and cliquewidth 4 graphs. In the case of binary tree $\cap$ partial grid graphs, the trouble is that recognizing partial grids is $N P$-complete [1]. However, for our purposes it suffices to observe that the class of binary tree graphs is of bounded cliquewidth and polynomial time recognizable [13]. In the case of chordal $\cap$ hamiltonian $\cap$ planar graphs, where the complexity of recognition is listed as "Unknown" in the ISGCI [13], it suffices to observe that this graph class has bounded treewidth (hence, bounded cliquewidth) and that there exists a linear time algorithm to decide if a graph has treewidth $\leq k$ [3]. In the case of thick tree graphs, where the complexity of recognition is again listed as "Unknown" in the ISGCI [13], we can observe that this graph class is contained in the class of bounded cliquewidth $\left(C_{n+4}\right.$, gem $)$-free graphs, which can trivially be recognized in polynomial time via its finite forbidden subgraph characterization. Finally, regarding the pathological case of the class of cliquewidth 4 graphs, we remark that while there exists a $O\left(n^{2} m\right)$ algorithm due to Corneil et al. [5] for deciding if a graph has cliquewidth $\leq 3$, the complexity of deciding if a graph has cliquewidth $\leq 4$ appears to be an open problem. Putting everything together yields the proposition at hand.

As an example of a conclusion that can be drawn from these results, we briefly remark that while it was previously understood that Bondy \& Jackson's non-existence conjecture [4] held for outerplanar graphs (which necessarily have at least two degree 2 vertices [8]), by Proposition 1 we have that any set of counterexamples for this conjecture cannot be contained in the class of $k$-outerplanar graphs for any fixed value of $k$ (unless $N P=R P)$. This is a consequence of the class of $k$-outerplanar graphs having bounded treewidth, which is again polynomial time detectable [3], or alternatively, a consequence of the combination of $k$-outerplanar graphs having bounded cliquewidth and being recognizable in polynomial time [2].

\section{References}

[1] Bhatt, S.N. and Cosmadakis, S.S.: The complexity of minimizing wire lengths in VLSI layouts, Inf. Process. Lett., Vol.25, No.4, pp.263-267 (1987).

[2] Bienstock, D. and Monma, C.L.: On the complexity of embedding planar graphs to minimize certain distance measures, Algorithmica, Vol.5, No.1-4, pp.93-109 (1990).

[3] Bodlaender, H.L.: A linear-time algorithm for finding treedecompositions of small treewidth, SIAM J. Comput., Vol.25, No.6, pp.1305-1317 (1996).

[4] Bondy, J.A. and Jackson, B.: Vertices of small degree in uniquely Hamiltonian graphs, J. Combin. Theory Ser. B, Vol.74, No.2, pp.265275 (1998).

[5] Corneil, D.G., Habib, M., Lanlignel, J.M., Reed, B. and Rotics, U. Polynomial-time recognition of clique-width $\leq 3$ graphs, Discrete Appl. Math., Vol.160, No.6, pp.834-865 (2012).

[6] Entringer, R.C. and Swart, H.: Spanning cycles of nearly cubic graphs, J. Combin. Theory Ser. B, Vol.29, No.3, pp.303-309 (1980).

[7] Espelage, W., Gurski, F. and Wanke, E.: How to solve NP-hard graph problems on clique-width bounded graphs in polynomial time, Proc. 27th International Workshop on Graph-Theoretic Concepts in Computer Science (WG), pp.117-128 (2001).

[8] Fleischner, H.: Uniquely Hamiltonian graphs of minimum degree 4, J. Graph Theory, Vol.75, No.2, pp.167-177 (2014).

[9] Ganian, R., Hliněný, P. and Obdržálek, J.: Clique-width: when hard does not mean impossible, Proc. 28th Annual Symposium on Theoret- 
ical Aspects of Computer Science (STACS), pp.404-415 (2011).

[10] Garey, M.R., Johnson, D.S. and Tarjan, R.E.: The planar Hamiltonian circuit problem is NP-complete, SIAM J. Comput., Vol.5, No.4, pp.704-714 (1976).

[11] Goedgebeur, J., Meersman, B. and Zamfirescu, C.T.: Graphs with few hamiltonian cycles, Math. Comp., Vol.89, pp.965-991 (2020).

[12] Haxell, P., Seamone, B. and Verstraete, J.: Independent dominating sets and Hamiltonian cycles, J. Graph Theory, Vol.54, No.3, pp.233244 (2007).

[13] de Ridder, H.N. et al.: Information System on Graph Classes and their Inclusions (ISGCI) (2001-2017), available from 〈http://www. graphclasses.org $\rangle$ (2016).

[14] Kelmans, A.K.: Constructions of cubic bipartite 3-connected graphs without Hamiltonian cycles, Amer. Math. Soc. Transl. (Ser. 2), Vol.158, pp.127-140 (1994).

[15] Liśkiewicz, M., Ogihara, M. and Toda, S.: The complexity of counting self-avoiding walks in subgraphs of two-dimensional grids and hypercubes, Theoret. Comput. Sci., Vol.304, No.1-3, pp.129-156 (2003).

[16] Royle, G. (https://mathoverflow.net/users/1492/gordon-royle): What is the smallest uniquely Hamiltonian graph with minimum degree at least 3?, MathOverflow, available from 〈https://mathoverflow.net/ q/255784 (version 2017-01-11).

[17] Sheehan, J.: The multiplicity of Hamiltonian circuits in a graph, Recent Advances in Graph Theory, Fiedler, M. (Ed.), pp.477-480 (1975).

[18] Thomason, A.G.: Hamiltonian cycles and uniquely edge colourable graphs, Ann. Discrete Math., Vol.3, pp.259-268 (1978).

[19] Thomassen, C.: Independent dominating sets and a second Hamiltonian cycle in regular graphs, J. Combin. Theory Ser. B, Vol.72, No.1, pp.104-109 (1998).

[20] Tutte, W.T.: On Hamiltonian circuits, J. London Math. Soc., Vol.s121, No.2, pp.98-101 (1946).

[21] Valiant, L.G.: Completeness for parity problems, Proc. 11th International Computing and Combinatorics Conference (COCOON), pp.1-8 (2005).

[22] Valiant, L.G. and Vazirani, V.V.: NP is as easy as detecting unique solutions, Theoret. Comput. Sci., Vol.47, pp.85-93 (1986).

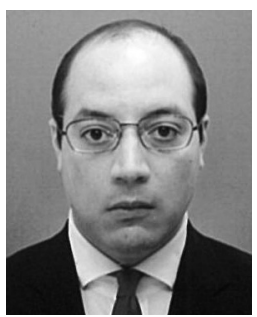

Robert D. Barish received his Ph.D. degree from the University of Tokyo in 2017, and is currently a project assistant professor at the University of Tokyo. His research interests include discrete mathematics, the complexity of counting and enumeration problems, differential privacy, and the application of these research areas in the physical sciences.

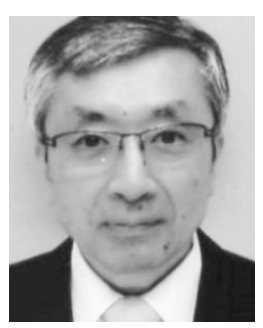

Akira Suyama held B.S. and Ph.D. degrees in Physics and Biophysics in 1977 and 1982 from the University of Tokyo. He was a professor of Department of Life Sciences and Institute of Physics, Graduate School of Arts and Sciences, the University of Tokyo. In 2020 he was named professor emeritus of the University of Tokyo. He remains interested in research on DNA computing and its application to Biological Sciences and Bio/Nanotechnology. 